D.T. Wickramasinghe, L. Ferrario, and G.V. Bicknell, eds.

\title{
The Disk Wind Model of the Broad Line Region of Active Galactic Nuclei
}

\section{J. Chiang and N. Murray}

Canadian Institute for Theoretical Astrophysics, 60 St George Street, Toronto, Ontario, M5S 3H8, Canada

\begin{abstract}
In a fashion similar to winds in $\mathrm{O}$ stars, the AGN disk winds may be responsible for the broad, blue-shifted absorption of resonance lines which characterize the Broad Absorption Line QSOs. The dynamical features of the disk wind may also account for many of the properties of the broad emission lines of AGN. Although the velocity field of the material responsible for the line emission is primarily that of the accretion disk, the presence of an accelerated radial wind influences the line formation so that single-peaked profiles are generic. Further, the recent reverberation mapping response of NGC 5548 is also qualitatively explained by these same radiative transfer effects.
\end{abstract}

\section{Introduction}

A defining characteristic of active galactic nuclei (AGNs) is the existence of broad, single-peaked emission lines in UV resonance transitions of ionized atoms such as CIv, Nv, Ovi, Sirv, MgII and Alin, as well as Ly $\alpha$. In approximately $10 \%$ of radio quiet QSOs, broad, deep blue-shifted absorption troughs in these lines are seen in addition to the broad line emission. Weymann et al. (1991) noted that the emission line features of these Broad Absorption Line QSOs (BAL QSOs) are otherwise very similar to the broad emission lines of ordinary QSOs. They concluded that the BAL QSOs and ordinary QSOs are intrinsically the same class of object and that the BAL phenomenon occurs when the observer line-of-sight passes through the absórbing material.

The similarity of the the BAL QSO line profiles to the P Cygni profiles found in O star spectra led Murray et al. (1995; hereafter M95) to consider a radiatively driven wind as the source of the observed broad absorption. Following the $\mathrm{O}$ star formalism of Castor, Abbott \& Klein (1975; hereafter CAK), M95 calculated the dynamics of a wind which is driven from the surface of an accretion disk. M95 derive an expression for the radial component of the streamline velocity structure which is identical in form to that found by CAK for O stars: $v_{r}=$ $v_{\infty}\left(1-\frac{r_{f}}{r}\right)^{\gamma}$, where $r_{f}$ is the radius of the streamline footpoint on the disk surface, the terminal velocity is of order the local escape velocity $v_{\infty} \sim v_{\text {esc }}$, and the exponent $\gamma \sim 1-1.3$.

This model made a number of predictions which were either consistent with existing observations or which have been shown to be true by later ones, including: smaller inner radii for the BLR than previously thought (cf. Krolik 
et al. 1991); large absorbing columns for BAL QSOs implying that these objects will also be X-ray quiet (cf. Green \& Mathur, 1996); the existence of very high ionization lines such as NeviII implying very high ionization parameters (cf. Hamann et al. 1995); a connection between the so-called warm absorbers in Seyfert galaxies and blue-shifted absorption features in the UV lines (Mathur, Elvis, \& Wilkes 1995).

\section{Emission Line Formation in a Disk Wind}

As in the case of $\mathrm{O}$ stars, the absorption features will be formed by resonant scattering of continuum radiation by ions in the wind. However, the bulk of the observed resonance line emission must be due to sources other than scattering since the equivalent widths of the emission features are typically much larger than would occur due to scattering alone. If collisional excitation or recombination are significant sources of line emission, then the bulk of the emission will originate where the density is the largest, i.e., at the wind footpoints along the surface of the disk.

The monochromatic specific luminosity for an emission line in a disk wind is

$$
L_{\nu}=\cos i \int_{\mathrm{disk}} d A l_{\mathrm{em}} k(r) S_{\nu}(r) \frac{1-e^{-\tau}}{\tau} \delta(\nu-\tilde{\nu}(r, \phi))
$$

where the absorption coefficient, $k(r)$, and the source function, $S_{\nu}(r)$ are, by the azimuthal symmetry of the disk, functions only of radius and are averaged vertically over the thickness, $l_{\mathrm{em}}$, of the emitting region (Murray \& Chiang 1996). The $\delta$-function insures that the integrand only makes a contribution to the line profile where the Doppler shift of the disk wind material matches the observed frequency $\nu$. The optical depth in the line at a point $(r, \phi)$ on the disk is given by

$$
\tau=\frac{1}{Q} \frac{k(r) c / \nu_{0}}{\sqrt{1+\left(l_{s} / l_{\mathrm{em}}\right)^{2}}}
$$

where the shear of the emitting material along the observer line-of-sight, $Q \equiv$ $|d \hat{v} / d \hat{n}|$, is approximately equal to

$$
Q \simeq \sin ^{2} i\left|\frac{\partial v_{r}}{\partial r} \cos ^{2} \phi-\frac{3}{2} \frac{v_{\phi}}{r} \sin \phi \cos \phi+\frac{v_{r}}{r} \sin ^{2} \phi\right|
$$

for high inclination systems ${ }^{1}$. The Sobolev length scale is $l_{s}=v_{\mathrm{th}} / Q$, where $v_{\text {th }} \sim 10^{6} \mathrm{~cm} \mathrm{~s}^{-1}$ is the thermal velocity

\section{Single-Peaked Line Profiles and Reverberation Mapping}

Near the wind footpoints, the radial component of the velocity is small compared with the azimuthal velocity, $v_{r} \sim 10^{7} \ll v_{\phi} \sim 10^{9} \mathrm{~cm} \mathrm{~s}^{-1}$. Hence, the lineemitting material will essentially be in circular orbits. However, the radial shear

${ }^{1}$ Additional terms for low inclination systems exist which depend on the $\hat{\theta}$-component of the disk wind velocity field. See Murray \& Chiang (1996) for details. 
associated with the wind is of the same order as the Keplerian shear of the disk itself, $\partial v_{r} / \partial r \simeq v_{\infty} / r \sim v_{\text {esc }} / r \sim v_{\phi} / r$. These large radial shears allow line photons to escape along directions with small projected velocities relative to the observer so that single- rather than double-peaked lines are produced.

This effect can be understood mathematically by considering the angular dependence of the integrand in Equation 1. At a given radius, it is proportional to $\left(1-e^{-\tau}\right) / \tau$, which is the directional escape probability $\beta$ in the Sobolev approximation (cf. Hamann, Korista \& Morris 1993). For an optically thick line, we have

$$
\beta \simeq \frac{1}{\tau} \propto\left|\left(v_{\infty} \cos \phi-v_{\phi} \sin \phi\right) \cos \phi\right| .
$$

In contrast, for a disk without the wind radial shears, we have $\beta \propto\left|v_{\phi} \sin \phi \cos \phi\right|$.

Figure 1 shows the disk surface brightness and corresponding line profiles seen by a high-inclination observer. On the left half, the familiar double-peaked profiles associated with disk motion are shown. (Horne \& Marsh 1986; see also Rybicki \& Hummer 1978). The factors $\cos \phi \sin \phi$ in $\beta$ for the disk-only case correspond to the low surface brightness (lighter pixels) areas where the projected velocities are near zero (cutting vertically through the image) and where the projected velocities are maximal (cutting horizontally). By contrast, the term proportional to $v_{\infty}$ in Equation 4 insures that the disk surface brightness is large (darker pixels) for $\phi \sim 0^{\circ}$ where the projected velocities are small. This yields the single-peaked profiles as shown in the right half of Figure 1.

In their analysis of the reverberation mapping observations of NGC 5548, Korista et al. (1995) found that the characteristic lag of the red-wing of the Civ line may be shorter than the other components of the line. Efforts to deconvolve the response function for various frequency components of the CIV line also yielded evidence that the red-wing responds stronger at earlier times (Wanders et al. 1995; Done \& Krolik 1996). Because the velocity field of an accretion disk is red-blue symmetric with respect to the observer, Done \& Krolik (1996) concluded that the timing response of the various components should also be redblue symmetric and hence dismissed it as a source for the broad lines. However, the anisotropic emission due the disk-wind shears changes this conclusion.

In the lower right panel of Figure 1, we have plotted over the disk wind surface brightness image, the iso-delay paraboloids for $1 / 2,5$ and 10 days (dashed lines) and the iso-frequency contours bounding the the blue (right side, solid lines) and red wings (left side). The hatched region on the right side corresponds to the blue response at early times, between $1 / 2$ and 5 days, and the hatched region on the left side corresponds to the red response at early times. We see that the emission anisotropy due to the disk wind can produce the stronger red wing response at early times similar to that seen in the reverberation mapping analyses of NGC 5548. A more complete discussion of these effects can be found in Chiang \& Murray 1996.

\section{Other Applications}

We are currently applying the disk wind formalism to single-peaked optical line profiles seen in SW Sextantis systems and other nova-like variables. 

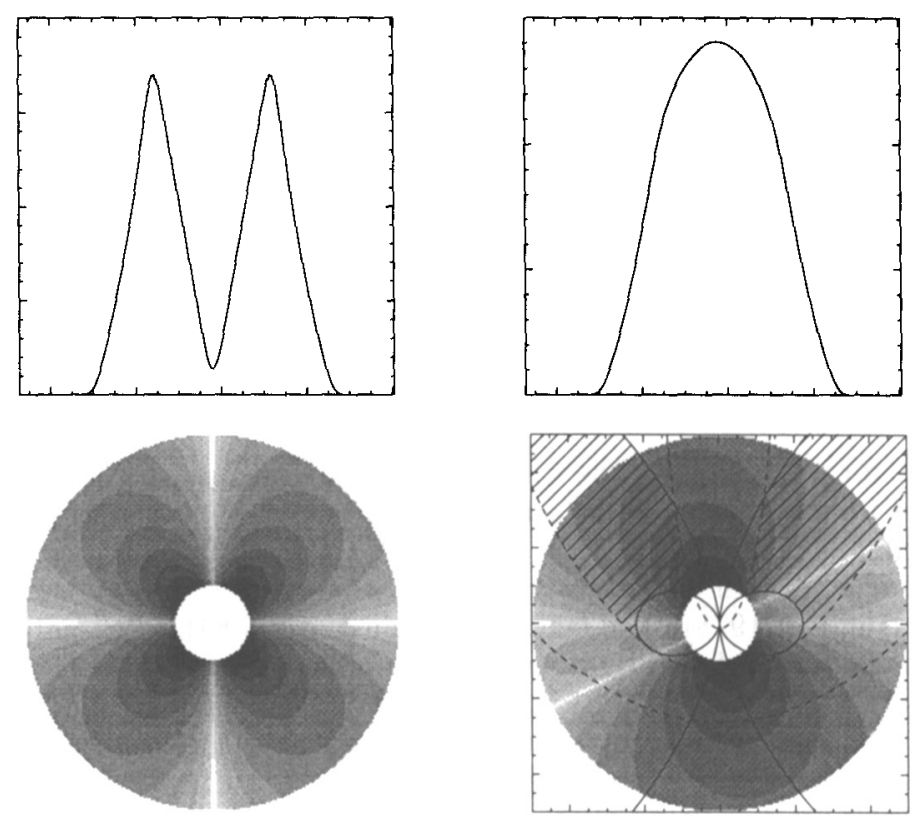

Figure 1.

Acknowledgments. This work was supported by NSERC of Canada and the Connaught Fund of the University of Toronto.

\section{References}

Castor, J. I., Abbott, D. C., \& Klein, R. I. 1975, ApJ, 195, 157

Chiang, J. \& Murray, N. 1996, ApJ, 466, 704

Done, C., \& Krolik, J. H. 1996, ApJ, 463, 144

Green, P. J., \& Mathur, S., 1996, ApJ, 462, 637

Hamann, F., et al. 1995, ApJ, 454, 688

Hamann, F., Korista, K. T., \& Morris, S. L. 1993, ApJ, 415, 541

Horne, K. \& Marsh, T. R. 1986, MNRAS, 218, 761

Korista, K., et al. 1995, ApJS, 97, 285

Krolik, J. H., et al. 1991, ApJ, 371, 541

Mathur, S., Elvis, M., \& Wilkes, B. 1995, ApJ, 452, 230

Murray, N., et al. 1995, ApJ, 451, 498 (M95)

Murray, N. \& Chiang, J. 1996, ApJ, in press

Rybicki, G. B., \& Hummer, D. G. 1978, ApJ, 219, 654

Wanders, I., et al. 1995, ApJ, 453, L87

Weymann, R. J., et al. 1991, ApJ, 373, 23 


\section{Discussion}

C. Heisler: Based on your model and fraction of BALs what is the opening angle you calculate?

$J$. Chiang: $\sim 10^{\circ}$ consistent with the observed fraction of BAL QSO's, relative to non-BAL radio quiet QSOs.

L. Maraschi: For what viewing angles do you recover a double peaked profile?

$J$. Chiang: Depending on the $\hat{\theta}$-component of the wind streamlines (which we haven't worked out in detail), double-peaked profiles may be produced at low inclinations $\left(i \leq 30^{\circ}\right)$. 